\title{
Some aspects of Routing Protocols at Wireless Sensor Networks
}

${ }^{1}$ Irfan Shaqiri ${ }^{2}$ Aristotel Tentov

${ }^{1}$ Ecolog International FZE Dubai, UAE irfantana@yahoo.com

${ }^{2}$ Faculty of Electrical Engineering and Information Technology-Skopje Macedonia toto@feit.ukim.edu.mk

\section{ABSTRACT}

In this paper we give an overview of some routing protocols which can improve the efficiency and scalability of wireless sensor networks. The Wireless Sensor Network (WSN) is a network consisting of ten to thousand small nodes with sensing, computing and wireless communication capabilities. WSN are generally used to monitor activities and report events, such as pollution parameters, healthcare issues, fire info etc. in a specific area or environment. It routs data back to the Base Station (BS). Data transmission is usually a multi-hop from node to node towards the BS. This type of networks is limited in power, computational and communication bandwidth. The main goal of all researchers is to find out the energy efficient routing protocol which will improve considerably networks resources in term of prolonging lifetime of sensor nodes. Also we highlight the various routing protocol with advantages and limitations as well.

\section{Indexing terms/Keywords}

WSN, Base Station, Protocol, Sensor, Node.

\section{Academic Discipline And Sub-Disciplines}

Computer Science and Engineering;

\section{SUBJECT CLASSIFICATION}

Wireless Sensors, Routing Protocols

\section{TYPE (METHOD/APPROACH)}

Theoreticaly method research

\section{INTRODUCTION}

Wireless Sensor Networks (WSN) are designed for environmental, health, military, agriculture monitoring and so on. The main goal of a wireless sensor node is to sense and collect data from a certain domain, process them and transmit it to the sink where the application lies. However, ensuring the direct communication between a sensor and the sink may force nodes to emit their messages with such a high power that their resources could be quickly depleted. Therefore, the collaboration of nodes to ensure that distant nodes communicate with the sink is a requirement. In this way, messages are propagated by intermediate nodes so that a route with multiple links or hops to the sink is established.

Considering the reduced capabilities of sensors, the communication with the sink could be initially formulated without a routing protocol. With this prerequisite, the flooding algorithm stands out as the simplest solution. In this algorithm, the transmitter broadcasts the data which are consecutively retransmitted in order to make them arrive at the intended destination.

However, its simplicity brings about significant drawbacks. Firstly, a deficiency is detected because nodes redundantly receive multiple copies of the same data message. Then, as the event may be detected by several nodes in the affected area,

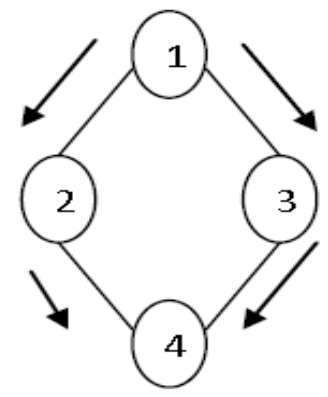

multiple data messages containing similar information are introduced into the network. Moreover, the nodes do not take into account their resources to limit their functionalities. One optimization relies on the gossiping algorithm [1]. Gossiping avoids deficiency (implosion Fig 1) as the sensor transmits the message to a selected neighbour instead of informing all its neighbours as in the classical flooding algorithm. However, overlap and resource blindness are still present.

\section{Fig. 1 Implosion (deficiencies) problem}

Besides, these disadvantages are highlighted when the number of nodes in the network increases. As a result of the deficiencies of the previous strategies, routing protocols become necessary in wireless sensor networks. Nevertheless, the inclusion of a routing protocol in a wireless sensor network is not a trivial task. 
One of the main limitations is the identification of nodes. Since wireless sensor networks are formed by a significant number of nodes, the manual assignation of unique identifiers becomes infeasible [2].

The use of potentially unique identifier such as the MAC (Medium Access Control) address or the GPS coordinates is not recommended as it forces a significant payload in the messages [3]. However, this drawback is easily overcome in wireless sensor networks since an IP address is not required to identify the destination node of a specific packet. In fact, attribute-based addressing fits better with the specificities of wireless sensor networks. In this case, an attribute such as node location and sensor type is used to identify the final destination.

Once nodes are identified, routing protocols are in charge of constructing and maintaining routes between distant nodes. The different ways in which routing protocols operate make them appropriate for certain applications. In the related literature, there are plenty of proposals concerning routing algorithms in wireless sensor networks.

In this paper we will describe the most important routing protocols that could be applied into wireless sensor networks and their characteristics.

\section{Algorithm Standards for Wireless Sensor Networks}

Sensor applications demand the communication of nodes to execute certain procedures or algorithms. In fact, three kinds of algorithms can be executed on wireless sensor networks [4]:

Centralized Algorithms: They are executed in a node that poses the knowledge of the whole network. These algorithms are quite rare because of the cost of transmitting the data to make the node know the status of the complete network.

Distributed Algorithms: The communication is supported by message-passing.

Local based Algorithms: The nodes use restricted data acquired from a close area. With this local information, the algorithm is executed in one node.

The algorithm standard is an important factor to take into account when deciding about the routing protocol to employ in the network. If localized algorithms are used, the routing protocol should reinforce and optimize the communication between neighbours. On the other hand, for centralized algorithms, combining the messages that simultaneously go the central node (even when they are generated by different sources) could be an advantage. The distributed algorithms should efficiently support the communication between any two pairs of nodes. Finally, local based algorithms depend on some solution that provides geographic coordinates, like GPS, making the solution more expensive.

\section{Design Constraints for Routing in Wireless Sensor Networks}

Due to the reduced computing, radio and battery resources of sensors, routing protocols in wireless sensor networks are expected to fulfil the following requirements [5]:

Autonomy: The assumption of a dedicated unit that controls the radio and routing resources does not stand in wireless sensor networks as it could be an easy point of attack. Since there will not be any centralized entity to make the routing decision, the routing procedures are transferred to the network nodes.

Energy Efficiency: Routing protocols should prolong network lifetime while maintaining a good grade of connectivity to allow the communication between nodes. It is important to note that the battery replacement in the sensors is infeasible since most of the sensors are randomly placed. Under some circumstances, the sensors are not even reachable. For instance, in wireless underground sensor networks, some devices are buried to make them able to sense the soil [6].

Scalability: Wireless sensor networks are composed of hundreds of nodes so routing protocols should work with this amount of nodes.

Resilience: Sensors may unpredictably stop operating due to environmental reasons or to the battery consumption. Routing protocols should cope with this eventuality so when a current-in-use node fails, an alternative route could be discovered.

Device Heterogeneity: Although most of the civil applications of wireless sensor network rely on homogenous nodes, the introduction of different kinds of sensors could report significant benefits. The use of nodes with different processors, transceivers, power units or sensing components may improve the characteristics of the network. Among other, the scalability of the network, the energy drainage or the bandwidth are potential candidates to benefit from the heterogeneity of nodes [7].

Mobility Adaptability: The different applications of wireless sensor networks could demand nodes to cope with their own mobility, the mobility of the sink or the mobility of the event to sense. Routing protocols should render appropriate support for these movements.

\section{Categorization of Routing Protocols in Wireless Sensor Networks}

Considering procedures which they have, routing protocols can be roughly categorized according to the following criteria. 


\section{Hierarchy Role of Nodes in the Network.}

In the flat schemes, all sensor nodes participate with the same role in the routing procedures. On the other hand, the hierarchical routing protocols classify sensor nodes according to their functionalities [8]. The network is then divided into groups or clusters. A leader or a cluster head is selected in the group to coordinate the activities within the cluster and to communicate with nodes outside the own cluster. The differentiation of nodes can be static or dynamic.

\section{Data Delivery Model}
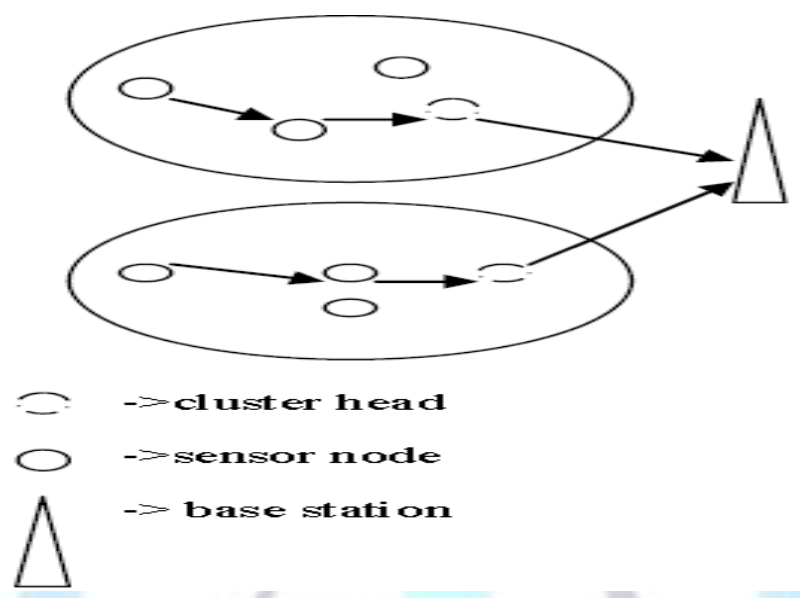

Fig. 2 Hierarchical routing

Depending on the application, data gathering and interaction in wireless sensor networks could be accomplished on several ways. The data delivery model indicates the flow of information between the sensor nodes and the sink [10]. The data delivery models are divided into the following classes: continuous, event-driven, query-driven or hybrid. In the continuous model, the nodes periodically transmit the information that their sensors are detecting at a pre-specified rate. In contrast, the query-driven approaches force nodes to wait to be demanded in order to inform about their sensed data. In the event-driven model, sensors emit their collected data when an event of interests occurs. Finally, the hybrid schemes combine the previous strategies so sensors periodically inform about the collected data but also response to queries. Additionally, they are also programmed to inform about events of interest.

\section{Optimization Techniques for Routing in WiRELess SEnsor Networks}

The particular characteristics of wireless sensor networks and their constraints have prompted the need for specific requirements to routing protocols. When compared to mobile ad hoc networks routing protocols, the algorithms in wireless sensor networks usually realize the following specifications:

Attribute-based: In these algorithms, the sink sends queries to certain regions and waits for the response from the sensors located in this area. Following an attribute-value scheme, the queries inform about the required data. The selection of the attributes depends on the application. An important characteristic of these schemes is that the content of the data messages is analyzed in each hop to make decisions about routing.

Energy Efficiency: Multiple routes can communicate a node and the sink. The aim of energy-aware algorithms is to select those routes that are expected to maximize the network lifetime. To do so, the routes composed of nodes with higher energy resources are preferred.

Data Aggregation: Since sensor nodes may generate significant redundant data, similar packets from multiple nodes can be aggregated so that the number of transmissions is reduced. Data aggregation is the combination of data from different sources according to a certain aggregation function, e.g., duplicate suppression, minima, maxima and average. This technique has been used to achieve energy efficiency and data transfer optimization in a number of routing protocols. Signal processing methods can also be used for data aggregation. In this case, it is referred to as data fusion where a node is capable of producing a more accurate output signal by using some techniques such as beamforming to combine the incoming signals and reducing the noise in these signals. Data collected in sensors are derived from common phenomena so nodes in a close area usually share similar information.

A way to reduce energy consumption is data aggregation. Aggregation consists of suppressing redundancy in different data messages. When the suppression is achieved by some signal processing techniques, this operation is called data fusion.

Addressing Scheme: Wireless sensor networks are consisted by a significant number of nodes so the manual assignation of unique identifiers is infeasible. The use of the MAC address or the GPS coordinates is not recommended as it introduces a significant payload [11]. However, network-wide unique addresses are not needed to identify the destination 
node of a specific packet in wireless sensor networks. In fact, attribute-based addressing fits better with the specificities of wireless sensor networks. In this case, an attribute such as node location and sensor type is used to identify the final destination. Concerning these identifiers, two different approaches have been proposed [13]. Firstly, the ID reuse scheme allows identifiers to be repeated in the network but keeping their uniqueness in close areas. In this way, a node knows that its identifier is unique in a k-hop neighborhood, being $\mathrm{k}$ a parameter to configure. On the other hand, the field-wide unique ID schemes guarantee that the identifiers are unique in the whole application. With this assumption, other protocols such as routing, MAC or network configurations can be simultaneously used.

Location-based: Once this technique is used, a node decides the transmission route according to the localization of the final destination and the positions of some other nodes in the network.

Multipath Communication: With this technique, nodes use multiple paths from an origin to a destination in the network. As multipath communications are intended to increase the reliability and the performance of the network, these paths should not share any link. Multipath communications can be accomplished in two ways: firstly, one path is established as the active communication routing while the other paths are stored for future need, i.e. when the current active path is broken. On the other hand, it is also possible to distribute the traffic among the multiple paths.

Quality of Service: The network application business and its functionalities prompt the need for ensuring a QoS (Quality of Service) in the data exchange. In particular, effective sample rate, delay bounded and temporary precision are often required. Satisfying them is not possible for all the routing protocols as the demands may be opposite to the protocol principles. For instance, a routing protocol could be designed to extend the network lifetime while an application may demand an effective sample rate which forces periodic transmissions and, in turn, periodic energy consumptions. In some applications, data should be delivered within a certain period of time from the moment it is sensed, otherwise the data will be useless [18]. Therefore, bounded latency for data delivery is another condition for time-constrained applications. However, in many applications, conservation of energy, which is directly related to network lifetime, is considered relatively more important than the quality of data sent. As the energy gets depleted, the network may be required to reduce the quality of the results in order to reduce the energy dissipation in the nodes and hence prolong the total network lifetime. So, energy-aware routing protocols are required to capture this requirement.

\section{CONCLUSIONS}

This paper reviews several different routing strategies for wireless sensor network. Therefore, routing protocols designed for WSN should be energy efficient as possible to prolong the life time of individual sensors.

Wireless Sensor Network technology extends numerous application domains and it is crucial that WSNs perform in reliable and robust manner. One of the major issues in the design of routing protocol for WSN is energy efficiency due to limited energy resources of sensors. Aiming at prolonging network lifetime, these protocols decides the best route according to three different metrics: battery availability, number of hops and link quality. The paper also includes some design constraints which should be considered always during process of modelling such sensor networks.

\section{REFERENCES}

[1] Zanaj, E.; Baldi, M.; Chiaraluce, F. Efficiency of the Gossip Algorithm for Wireless Sensor Networks. In Proceedings of the 15th International Conference on Software, Telecommunications and Computer Networks (SoftCOM), SplitDubrovnik, Croatia, September, 2007.

[2] Zhou, H.; Mutka, M.W.; Ni, L.M. Reactive ID Assignment for Sensor Networks. In Proceedings of the 2nd IEEE International Conference on Mobile Ad-hoc and Sensor Systems (MASS), Washington, DC, USA, November, 2005.

[3] Lin, J.; Liu, Y.; Ni, L.M. SIDA: Self-organized ID Assignment in Wireless Sensor Networks. In Proceedings of the 4th IEEE International Conference on Mobile Ad-hoc and Sensor Systems Conference (MASS), Pisa, Italy, October, 2007.

[4] Boukerche, A.; Nakamura, E.F.; Loureiro, A.F. Algoritms for Wireless Sensor Networks. In Algorithms and Protocols for Wireless Sensor Networks; Boukerche, A., Ed.; John Wiley \& Sons: Hoboken, NJ, USA, 2009.

[5] Akkaya, K.; Younis, M. A Survey on Routing Protocols for Wireless Sensor Networks. Ad Hoc Netw. 2005, 3, 325349.

[6] Akyildiz, I.; Pompili, D.; Melodia, T. Underwater Acoustic Sensor Networks: Research Challenges. Ad Hoc Netw. 2005, 3, 257-279.

[7] Braginsky, D.; Estrin, D. Rumor Routing Algorithm for Sensor Networks. In Proceedings of the First ACM International Workshop on Wireless Sensor Networks and Applications (WSNA), Atlanta, GA, USA, September, 2002; pp. 22-31.

[8] Yao, Y.; Gehrke, J. The Cougar Approach to In-Network Query Processing in Sensor Networks. SIGMOD Rec. 2002, 31, 9-18.

[9] Sadagopan, N.; Krishnamachari, B.; Helmy, A. The ACQUIRE Mechanism for Efficient Querying in Sensor Networks. In Proceedings of the First IEEE International Workshop on Sensor Network Protocols and Applications (SNPA), Anchorage, AK, May, 2003; pp. 149-155. 
[10] Rao, A.; Ratnasamy, S.; Papadimitriou, C.; Shenker, S.; Stoica, I. Geographic Routing without Location Information. In Proceedings of the Ninth Annual International Conference on Mobile Computing and Networking (MOBICOM), San Diego, CA, USA, September, 2003; pp. 96-108.

[11] Seada, K.; Helmy, A. Geographic Protocols in Sensor Networks; Technical Report 04-837; Computer Science Department, University of Southern California: San Diego, CA, USA, 2008.

[12] Seada, K.; Zuniga, M.; Helmy, A.; Krishnamachari, B. Energy-Efficient Forwarding Strategies for Geographic Routing in Lossy Wireless Sensor Networks. In Proceedings of the Second International Conference on Embedded Networked Sensor Systems, Baltimore, MD, USA, November, 2004; pp. 108-121.

[13] He, T.; Stankovic, J.A.; Lu, C.; Abdelzaher, T.F. SPEED: A Stateless Protocol for Real-Time Communication in Sensor Networks. In Proceedings of the 23rd International Conference on Distributed Computing Systems (ICDCS), Providence, RI, USA, May, 2003; pp. 46-55.

[14] Fang, Q.; Gao, J.; Gubias, J. Locating and Bypassing Routing Holes in Sensor Networks. In Proceeding of the 23rd Conference of the IEEE Communications Society (INFOCOM), Hong Kong, China, March, 2004; pp. $2458-2468$.

[15] Xu, Y.; Heidemann, J.; Estrin, D. Geography-informed Energy Conservation for Ad Hoc Routing. In Proceedings of the Seventh Annual International Conference on Mobile Computing and Networking (MOBICOM), Rome, Italy, July, 2001; pp. 70-84.

[16] Rabiner, W.; Kulik, J.; Balakrishnan, H. Adaptive Protocols for Information Dissemination in Wireless Sensor Networks. In Proceedings of the Fifth Annual International Conference on Mobile Computing and Networking (MOBICOM), Seattle, WA, USA, August, 1999; pp. 174-185.

[17] Heinzelman, W.B.; Chandrakasan, A.P.; Balakrishnan, H. An Application-Specific Protocol

[18] Barbancho, J.; León, C.; Molina, F.J.; Barbancho. A. A New QoS Routing Algorithm based on Self-Organizing Maps for Wireless Sensor Networks. Telecommun. Syst. 2008, 36, 73-83.

\section{Author's Biography}

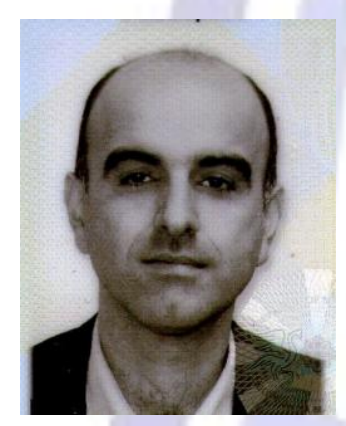

Irfan Shaqiri received his M.Sc. at the Computer Science and Engineering Department, University Ss Cyril and Methodius-Skopje, Macedonia and is currently working toward gaining his Ph.D. degree in the same institution. He is an IT Manager in the Department of Information Technologies at Ecolog-International FZE Dubai

His research field of interests are Wireless Sensors, Routing Protocols, Network Security etc. 